\title{
Reporte clínico: Registro piezográfico de la zona neutra, en un paciente con espasmos musculares involuntarios
}

\author{
Clinical report: Piezographyc record of the neutral \\ zone in a complete edentulous patient with involuntary \\ muscle spasms
}

\author{
Astorga $\mathrm{E}^{1}$, Lee $\mathrm{X}^{2}$, Vergara $\mathrm{C}^{3}$
}

\begin{abstract}
RESUMEN
Toda función del sistema estomatognático involucra la acción sinérgica de la musculatura paraprotética, representada por estructuras complejas tales como lengua, labios, mejillas y piso de boca. Al rehabilitar a través de prótesis completas no siempre se considera la influencia de la dinámica muscular, y menos aún cuando alguna de estas estructuras presenten alguna patología, por ejemplo, el compromiso del nervio facial que genera espasmos musculares involuntarios. En tal situación, la ubicación de los dientes y la forma de las superficies pulidas pueden llegar a ser decisivos entre éxito o fracaso de la rehabilitación. Se sabe que los implantes oseointegrados proveen retención, soporte y estabilidad, sin embargo existen situaciones especiales en que no es posible acceder a este tipo de tratamiento, tanto por razones médico quirúrgicas o costos asociados. En este estudio se ha utilizado un registro Piezográfico de la Zona Neutra en un paciente con compromiso del nervio facial que genera espasmos musculares involuntarios del lado afectado, obteniéndose una rehabilitación protésica basada en la apropiada relación entre superficie protésica externa y tejidos circundantes, mejorando requisitos funcionales tales como retención y estabilidad. Se ha utilizado el sistema de tomografía computarizada Cone Beam, con el objetivo de evidenciar el registro piezográfico. Los resultados obtenidos con estas prótesis piezográficas demuestran ser superior en factores como comodidad y fonoarticulación pero inferior en eficiencia masticatoria comparada con la técnica convencional.
\end{abstract}

Rev. Clin. Periodoncia Implantol. Rehabil. Oral Vol. 6(3); 134-137, 2013.

Palabras clave: Zona neutra, piezografía, prótesis totales.

\section{ABSTRACT}

Every function of the stomatognathic system involves the synergistic action of the musculature around the prosthesis, consisting of complex and individual structures such as tongue, lips, cheeks and mouth floor. When planning and making complete prostheses, the muscular dynamic is not always considered. This can generate instability of the device during function, the location of the artificial teeth is made arbitrarily on the top and middle parts of the flange, and the configuration of the polished areas is made according to mechanistic concepts. This factor takes more relevance in patients with severe atrophy of their residual flanges, where the prosthetic stability can be a decisive factor between the success or failure of the rehabilitation. It is known that the osseointegrated implants provide retention, support and stability. However, there are special situations in which it is not possible to access to this type of treatment, both for medical surgical reasons or associated costs. In this study, a Piezographyc record of the Neutral Zone was used in a patient with facial nerve involvement generating involuntary muscle spasms of the affected side. A prosthetic rehabilitation based on the appropriate relation between external prosthetic surface and surrounding tissues was obtained, improving functional requirements such as retention and stability. The computerized tomographic Cone Beam system was used, with the objective to show the piezographic record. The results obtained with these piezographic prostheses prove to be superior in factors like comfort and speech articulation, but inferior in masticatory efficiency, where the conventional technique is still better.

Rev. Clin. Periodoncia Implantol. Rehabil. Oral Vol. 6(3); 134-137, 2013.

Key words: Neutral zone, piezography, total prosthesis.

\section{INTRODUCCIÓN}

Zona Neutra en Rehabilitación de Pacientes Desdentados Completos La zona neutra ha sido definida como aquella área o espacio potencial de la cavidad oral edéntula, donde las fuerzas musculares de la lengua, los labios y las mejillas se neutralizan. Este espacio no necesariamente se ubica sobre la cresta del reborde, o hacia lingual o bucal de él. Su ubicación dependerá de la musculatura protésica, la cual varía entre los pacientes, y no necesariamente corresponde con la posición mecánica de los dientes protésicos, debido principalmente a los diversos patrones de reabsorción del reborde alveolar en los diferentes segmentos maxilares. También, se debe destacar que existen diferencias individuales que comienzan a establecerse desde la erupción de los dientes naturales, los cuales están expuestos a influencias ambientales conocidas $^{(1,2)}$ (Figura 1).

Los tejidos blandos que conforman las superficies interna y externa de la prótesis, pueden afectar e influenciar la estabilidad de ellas. Imprimir dicha zona permitirá determinar adecuadamente los bordes periféricos, posición dentaria y contornos externos ${ }^{(3,4)}$. Cabe destacar que el registro de las fuerzas desarrolladas a partir de la contracción

1. Cirujano Dentista. Bachiller con mención en Ciencias Naturales y Exactas. Diplomado en Rehabilitación del Paciente Desdentado Completo por Métodos Convencionales e Implantes Óseointegrados. Facultad de Odontología, Universidad de Chile. Chile. Académico. Departamento de Prótesis. Facultad de Odontología, Universidad de Chile. Chile.

2. Cirujano Dentista. Especialista en Rehabilitación Oral. Facultad de Odontología, Universidad de Chile. Chile. Magíster en Educación en Ciencias de la Salud. Facultad de Medicina, Universidad de Chile. Chile. Académico. Departamento de Prótesis. Facultad de Odontología, Universidad de Chile. Chile. 3. Cirujano Dentista. Especialista en Rehabilitación Oral y en Ortopedia y Ortodoncia Dento Máxilo Facial. Facultad de Odontología, Universidad de Chile. Chile. Académico. Departamento de Prótesis. Facultad de Odontología, Universidad de Chile. Chile. 
muscular durante la masticación, el habla o la deglución favorecerá la estabilidad protésica.

Existen condiciones del paciente que favorecen la inestabilidad protésica, podemos mencionar ${ }^{(5,6,7)}$ : Enfermedad de Parkinson, accidente cerebrovascular, esclerodermia, prominencia del canal mentoniano, pacientes sometidos a resecciones quirúrgicas de la mandíbula, glosectomía parcial, daño del nervio motor de la lengua, pacientes con pobre control neuromuscular, terapia miofuncional, alargamiento quirúrgico de la banda del buccinador, vestibuloplastía, cirugía ortognática, espasmos hemifacial, entre otros. Este útlimo es un transtorno motor caracterizado por la contracción incontrolada, intermitente y espasmódica o tónica de los musculos de una hemicara(7).

La zona neutra es, además, un área dinámica, modificada en el tiempo, especialmente por la continua reabsorción del hueso del reborde residual y por cambios en las fuerzas de la musculatura paraprotética(7-10).

Se ha determinado que la reabsorción del reborde residual es mayor en el hueso mandibular, dejando menor cantidad de tejido para dar retención y soporte a las prótesis inferiores aumentando la dificultad en su confección. Debido a esto, las prótesis mandibulares suelen ser menos usadas por los pacientes por provocar dolor e inestabilidad ${ }^{(11,12)}$. Además, en una reabsorción de reborde residual avanzada, donde el vestíbulo se encuentra muy disminuido, se puede llegar hasta la zona de las inserciones musculares e inclusive quedar sobre el reborde residual, como sucede en la zona anterior con el músculo mentoniano, en cuyo caso la zona neutra sufre un desplazamiento en la zona anterior hacia lingual ${ }^{(13)}$

Por lo tanto, un factor importante a considerar es el tiempo de edentulismo: Si este es corto, se describe que los dientes pueden ser colocados sobre el reborde de acuerdo a la técnica clásica, o usando la zona neutra; en cambio si es un tiempo largo de edentulismo, la zona neutra debiera registrarse.

Con respecto a los músculos y su relación con las prótesis completas la función y actividad musculares actúan sobre ellas y, por ende, afectan la estabilidad protésica en boca. Por ello es importante la ubicación de los dientes artificiales dentro de un área que sea compatible con las fuerzas musculares para obtener una influencia positiva en la estabilidad final del dispositivo $0^{(3,4,14)}$.

Las principales fuerzas musculares que actúan en las prótesis completas son las generadas por (Figura 2):

1. Buccinador: Cumple un rol importante en la determinación de la zona neutra y depende de la posición de los arcos dentarios y del contorno de la base protésica para su función efectiva ${ }^{(3,4)}$.

2. Modiolo: Impide el escape del alimento por la comisura cuando está siendo desmenuzado por los premolares y molares, por lo tanto, se debe asegurar su libre funcionamiento para no afectar la estabilidad de la prótesis inferior y ello se logra reduciendo el ancho buco lingual de la superficie externa de la prótesis ${ }^{(3,4)}$.

3. Orbicular de los Labios y Mentoniano: Determinan la posición de los dientes anteroinferiores en conjunto con los movimientos e interacción con la lengua Por la reabsorción del reborde residual avanzada la inserción muscular del mentoniano puede ubicarse muy cercano al reborde desplazando la zona neutra, que según Lammie es hacia lingual. En un estudio realizado por FM Fahmi et al. (1992), encontraron que en pacientes con desdentamientos de más de dos años, la zona neutra se ubica hacia vestibular en aproximadamente $2 \mathrm{~mm}^{(14)}$; lo que implica la ubicación de los dientes antero inferiores hacia vestibular del reborde alveolar, sin embargo no se debe olvidar la fonoarticulación y estética del paciente ${ }^{(3,4)}$.

4. Lengua: Durante la función el factor crítico está dado por la posición de los dientes anteriores y posteriores respecto a la ubicación lingual, al no ser considerada y disponer por ejemplo, los dientes hacia lingual, se produciría una disminución en el espacio para la lengua provocando inestabilidad en la prótesis. Además, la lengua tiende a expandirse en bocas desdentadas, determinando el desplazamiento de la posición buco lingual de la zona neutra ${ }^{(3,4,15)}$.

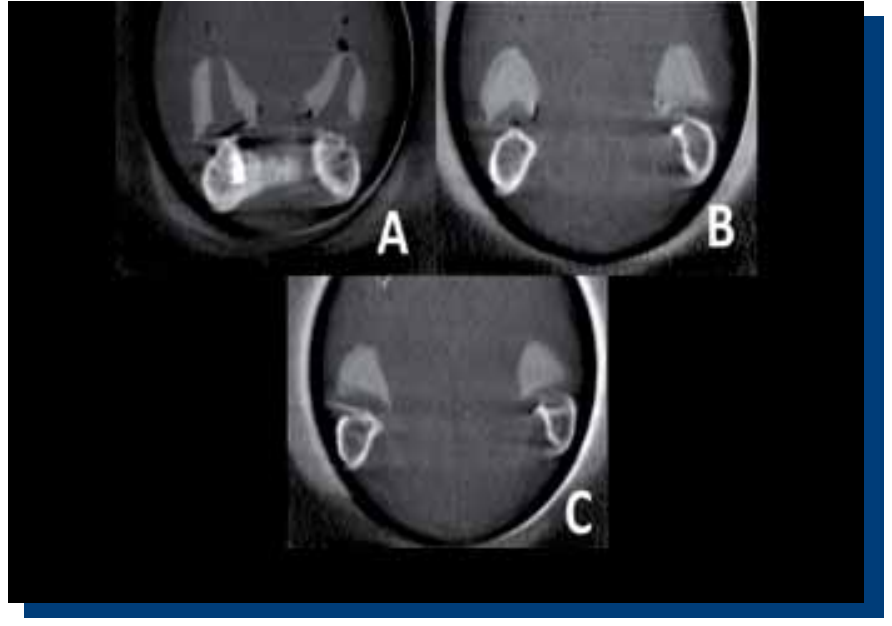

Figura 1. Cortes imagenológicos transversales de la zona neutra. (A) Corte anterior (B) Corte zona premolares. (C) Corte zona molares.

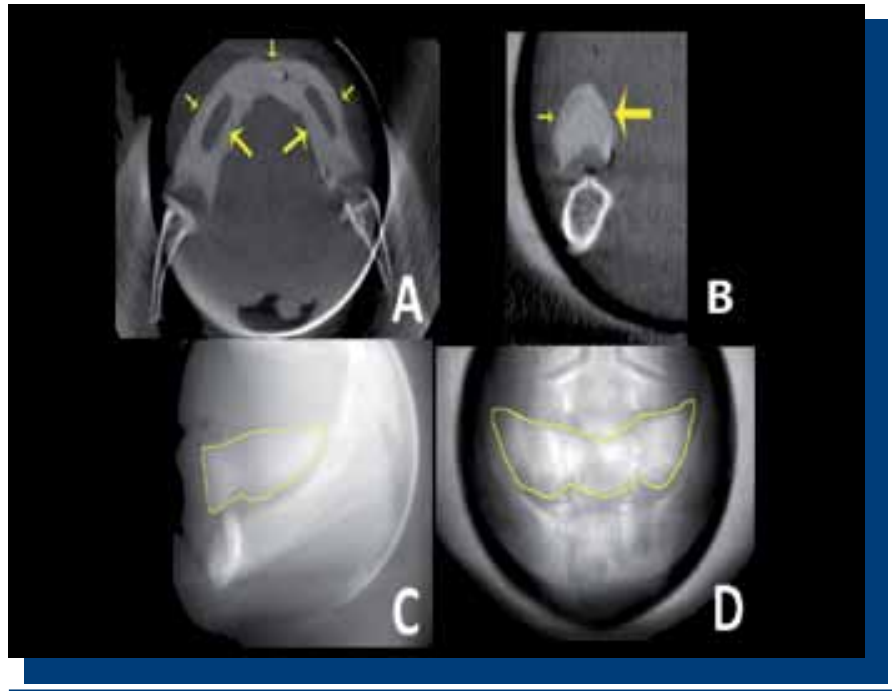

Figura 2. (A) Vista oclusal del registro piezográfico. Las flechas indican los vectores de fuerza muscular. (B) Corte transversal del lado izquierdo del sector molar. Las flechas indican los vectores de fuerza muscular. (C) Vista de perfil. (D) Vista frontal.

\section{Registro de la Zona}

La técnica piezográfica registra la forma que debe tener la superficie externa de la prótesis y la posición de las piezas dentarias a través de la función muscular de los músculos paraprotéticos, permitiendo confeccionarla en la zona neutra, y mejorando la propiocepción de paciente desdentado ${ }^{(16,17,18)}$.

Para determinar la zona neutra se utiliza un material de impresión, con consistencia de pasta, el cual gracias a las presiones ejercidas por las paredes de este espacio potencial durante el desarrollo de ciertas funciones, este modelado transforma la zona neutra en un espacio piezográfico ${ }^{(19)}$

\section{Materiales de Registro}

Como el registro piezográfico depende de la función muscular se debe utilizar un material que pueda ser moldeable por la actividad muscular, ya que se busca determinar la forma de los arcos y contorno del cuerpo de la prótesis.

Se han propuesto muchos materiales para realizar la técnica, dentro de los cuales se encuentran: Compuesto de modelar, silicona, acondicionador de tejidos y materiales resilientes ${ }^{(20)}$.

El material, por lo tanto, debe cumplir con requisitos para obtener un registro fiel de la zona neutra, siendo lo más importante la plasticidad para modelar las fuerzas musculares y que permita adicionar material para obtener el ancho adecuado. 
Además, se debe confeccionar una placa piezográfica de acrílico de autopolimerización (Figura 3A) que permite ubicar el material en la zona del rodete de oclusión y así registrar las fuerzas musculares ${ }^{(21)}$.

La placa piezográfica debe cumplir dos funciones:

1. Mantener la dimensión vertical oclusal.

2. Dar soporte al material de impresión para la zona neutra.

Esto se consigue al colocar en la zona oclusal de la placa piezográfica topes en el área de los premolares, preocupándose de no afectar la función de los labios, las mejillas y la lengua para obtener un registro fiel.

\section{Técnicas de Registro}

Piezografía en Fonación

Durante la fonoarticulación se abre levemente la boca para que se produzcan fuerzas de diferente magnitud y dirección, con componentes verticales y horizontales que actuarán directamente en las prótesis ${ }^{(22)}$.

Si se eligen bien los fonemas modelantes, así como su intensidad, es posible obtener una actividad muscular sincronizada que garantiza un modelado eficaz obteniendo unas prótesis estables y confortables, ya que su volumen queda bien adaptado a las reducidas superficies de sustentación de las zonas edéntulas (Figura 3B y C).

El estudio realizado por Makzoumé en 2004, encontró que en esta técnica hay diferencias significativas en la zona de los premolares, ubicándose hacia lingual con respecto a la técnica clásica de ordenamiento dentario. Además, el espacio protético obtenido con la técnica fonética, es más estrecho y se ubica hacia lingual al compararla con la técnica de la deglución, la cual tiene una gran actividad lingual, lo que genera mayor desplazamiento de la zona piezográfica hacia vestibular, generando zonas voluminosas en el registro, que luego tienen la tendencia a ser desestabilizadas, especialmente cuando la cinta vestibular entra en actividad (Figura 3B y C).

Teniendo en cuenta que la obtención de la zona neutra, a través de un registro piezográfico, está especialmente indicado en aquellos pacientes que presentan condiciones locales o generales que favorecen la inestabilidad protésica, es que el propósito de este estudio es describir el registro piezográfico de la zona neutra en un paciente desdentado completo con compromiso de nervio facial que genera espasmo musculares involuntarios, evidenciando dicho registro en la cavidad oral mediante el sistema de tomografía computarizada Cone Beam.

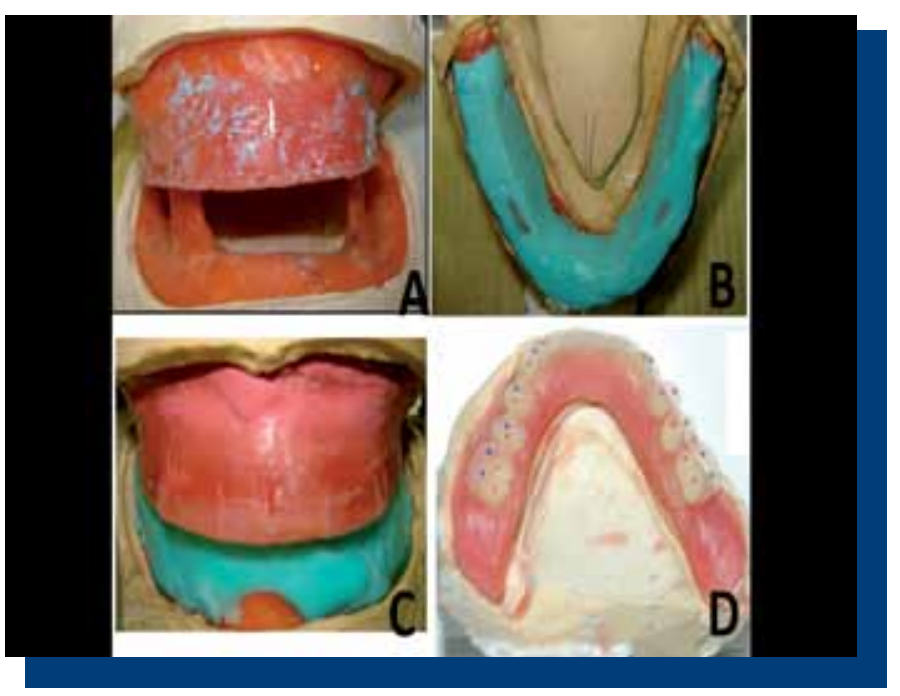

Figura 3. (A) Placa piezográfica. (B) Vista oclusal de un registro piezográfico. (C) Vista frontal. (D) Comparación entre convencional y piezográfica (nótese la distancia y ubicación entre puntos azules y rojos).

\section{MATERIAL Y MÉTODO}

Se seleccionó un paciente de 75 años género masculino, ASA II diabético compensado, desdentado completo superior e inferior, con reabsorción de reborde tipo VI de Attwood con espasmos musculares involuntarios debido a un accidente automovilístico. El paciente fue sometido a cirugía de dos implantes mandibulares en la zona de las piezas 22 y 27 para ser posteriormente rehabilitado con una sobredentadura sobre implantes inferior, en la Clínica de Prótesis Totales, en la Facultad de Odontología de la Universidad de Chile. El paciente fue instruido respecto de la técnica piezográfica y del estudio imagenológico Cone Bean y posteriormente el paciente firma los respectivos consentimientos informados utilizados rutinariamente en la asignatura.

Se efectuó una impresión preliminar para confeccionar una placa piezográfica de acrílico autopolimerizable marca Marche con topes oclusales (Figura 3A).

Para el registro piezográfico se preparó una pasta de silicona de consistencia pesada y mediana, marca Speedex ${ }^{\circledR}$, en una proporción de $7 \mathrm{~cm}$ de mediana / $1 / 2$ porción de pesada (Figura 3B y C).

En cuanto a la posición del paciente, esta fue sentado en el sillón dental cómodamente y en posición habitual.

Se registró la zona neutra con la técnica de fonación, previa instrucción de paciente. Se utilizaron los fonemas: SOSO, SIS para la formación de un muro posterior, y ME, PE, TE y DE para la formación de un muro anterior del registro piezográfico. El plano de oclusión se determinó con los bordes de la parte media de la lengua al pronunciar el fonema $\mathrm{E}$, y con el fascículo medio del buccinador.

Se registró fotográficamente la secuencia del procedimiento y por último se procedió al registro imagenológico computarizado Cone Beam, para evidenciar en la cavidad oral el registro piezográfico, según protocolos del Servicio de Radiología de la Facultad de Odontología.

\section{RESULTADOS}

El reporte clínico con el registro piezográfico realizado a un paciente desdentado completo con espasmos musculares involuntarios y evidenciado con cone beam, devela lo siguiente: Las flechas de color amarillo de la Figura 2A indican los componentes de fuerza realizados por el orbicular de los labios, mentoniano, grupo muscular que conforma el modeolo y buccinador por vestibular y la lengua en la zona lingual. Nótese la diferencia entre el sector anterior y posterior, siendo de un menor grosor en la zona anterior y mayor grosor en el lado izquierdo del paciente. En la Figura $2 \mathrm{~B}$ se observan flechas amarillas, que indican un mayor vector de fuerza en la zona lingual por el desplazamiento del registro piezográfico hacia la zona vestibular al comparar con la zona más alta del reborde. En la Figura 2D, al comparar el lado izquierdo con el lado derecho, se observa un mayor desplazamiento del registro en el lado derecho del paciente, en relación al lado de mayor actividad muscular.

En una vista de perfil de la tomografía con el registro piezográfico (Figura $2 \mathrm{C}$ ) se observa la relación con el labio del paciente después del modelado.

Al observar cortes transversales de la tomografía con el registro piezográfico, a niveles anterior, premolar y molar (Figura 1A, B y C), se evidencia que: En el sector anterior existe una discrepancia entre la zona más alta del reborde y el registro piezográfico, con un mayor desplazamiento hacia vestibular de este último. En el sector de los premolares hay mayor coincidencia en el lado derecho entre el registro piezográfico y la zona más alta del reborde, a diferencia del lado izquierdo donde hay una pequeña variación hacia lingual. En la zona molar en el lado derecho del paciente se observa un desplazamiento hacia vestibular del registro piezográfico, en relación a la actividad muscular y en el lado izquierdo existe un desplazamiento hacia lingual con respecto a la zona más alta del reborde en relación a los espasmos musculares involuntarios.

\section{DISCUSIÓN}

La técnica de registro piezográfico de la zona neutra permitió realizar un registro funcional de la musculatura paraprotética, 
obteniéndose el tercer nivel de funcionalidad, y mejorando de esta forma la estabilidad del aparato protésico. La realización de la técnica involucró un mayor tiempo clínico, entrenamiento previo del paciente y capacitación al técnico dental para la fase de terminación y pulido de la prótesis.

En este caso clínico, evidenciado a través de la técnica imagenológica Cone Beam, se pudieron determinar diferencias en el registro obtenido en los sectores anteriores, premolares y molares, presentando mayores diferencias en el sector molar.

Al comparar el lado izquierdo y derecho del paciente, hay discrepancia de en el lado izquierdo. Esta diferencia se justifica por la alteración del tono muscular del paciente, quien presenta espasmos musculares producto de un daño en el nervio facial consecutivo al traumatismo previamente descrito, traduciéndose en que el registro piezográfico haya sufrido un desplazamiento hacia lingual.

En relación a la discrepancia del lado derecho se evidencia el desplazamiento descrito por otros autores ${ }^{(2,7,13,23,24)}$ por la acción muscular donde la lengua genera el mayor componente de fuerza.

\section{CONFLICTOS DE INTERÉS}

Los autores declaran no tener conflictos de interés.

\section{REFERENCIAS BIBLIOGRÁFICAS}

1. Agarwal S, Gangadhar P. A simplified approach for recording neutral zone. J Indian Prosthodont Soc, 2010; 10(2): 102-104.

2. Hina Z, Raja M, Nasir S. Neutral zone dentures versus conventional dentures diverse edentulous periods. Biomedica, 2009; 25: 136-145.

3. Gahan MJ, Walmsley AD. The neutral zone impression revisited. Br Dent J, 2005; 198(5): 269-272.

4. Beresin VE, Shiesser FJ. The neutral zone in complete dentures. J Prosthet Dent, 2006; 95(2): 93-101.

5. Alfano SG, Leupold RJ. Using the neutral zone to obtain maxillomandibular relationship records for complete denture patients. J Prosthet Dent, 2001; 85(6): 621-623.

6. Ohkubo C, Hanatani S, Hosoi T, Mizuno Y. Neutral zone approach for denture fabrication for a partial glossectomy patient: A chinical report. J Prosthet Dent, 2000; 84(4): 390-393.

7. Ikebe K, Okuno I, Nokubi T. Effect of adding impression material to mandibular denture space in piezography. J Oral Rehabil, 2006; 33: 409-415.

8. Barrenas L, Ödman P. Myodynamic and conventional construction of complete dentures: A comparative study of comfort and function. J Oral Rehabil, 1989; 16: 457-465.

9. Weinberg LA. Tooth position in relation to the denture base foundation. J Prosthet Dent, 1958; 8(3): 398-405

10. Alajbeg AZ, Valentic M, Alajbeg I, Illes T, Celebic A. The influence of dental status on masticatory muscle activity in elderly patients. Int J Prosth, 2005; 18(4): 333-338.

11. Indirapadmaja B, Prabhakar P, Reddy R, Kumar A. Neutral zone in complete denture. Dentaires Revista, 2009; 1(1): 5-9.
12. Pekkan G, Hekimoglu C, Sahin N. Rehabilitation of a marginal mandibulectomy patient using a modified neutral zone technique: A case report. Braz Dent J, 2007; 18(1): 83-86.

13. Lynch C, P Finbarr A. Overcoming the unstable mandibular complete denture The neutral zone impression technique. Dent Update, 2006; 33: 21-26.

14. Fahmy FM. The position of the neutral zone in relation to the alveolar ridge. $J$ Prosthet Dent, 1992; 67(6): 805-809.

15. Miller WP, Monteith B, Heath MR. The effect of variation of the lingual shape of mandibular complete dentures on lingual resistance to lifting forces. Gerodontology, 1998; 15(2): 113-119.

16. Shekar C. Manegement of a severely resorbed mandibular ridge with the neutral zone technique. Contemp Clin Dent, 2010; 1(1): 36-39.

17. Rao S, Chowdhary R, Mahoorkar S. A systematic review of impression technique for conventional complete denture. J Indian Prosthodont Soc, 2010; 10(2): 105-111.

18. Gahan M. The neutral zone impression revisited. Br Dent J, 2005; 198: 269- 272

19. Brill N, Tryde G, Cantor R. The dynamic nature of the coger denture space. Prosthet, 1965; 15(3): 401-417.

20. Klein P. Piezología, piezografía y prótesis removible. Internacional College of Dentists Section Tour, 1997; 5(1): 17-23.

21. Agarwal S, Gangadhar P. A simplified approach for recording neutral zone. J Indian Prosthodont Soc, 2010; 10(2): 102-104.

22. Beresin S. The neutral zone in complete and partial dentures. Mosby. St. Louis. 1978 23. Fahmy FM. The position of the neutral zone in relation to the alveolar ridge. $J$ Prosthet Dent, 1992; 67(6): 805-809.

24. Makzoumé JE. Morphologic comparison of two neutral zone impression techniques: A pilot study. J Prosthet Dent, 2004; 92(6): 563-568. 